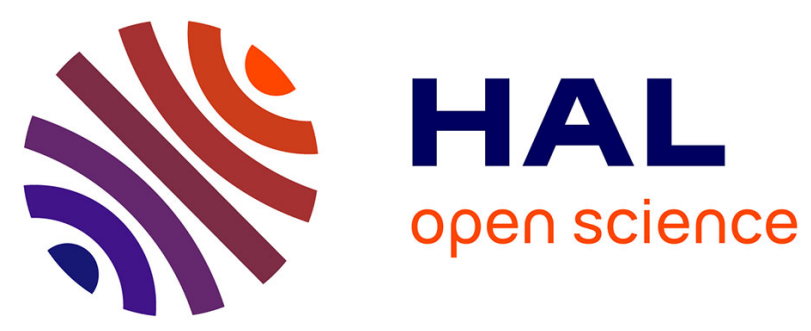

\title{
Turbulence and columnar vortex formation through inertial-wave focusing
}

\author{
Matias Duran-Matute, Jan-Bert Flór, Fabien S. Godeferd, Cl ément
}

Jause-Labert

\section{- To cite this version:}

Matias Duran-Matute, Jan-Bert Flór, Fabien S. Godeferd, Cl ément Jause-Labert. Turbulence and columnar vortex formation through inertial-wave focusing. Physical Review E: Statistical, Nonlinear, and Soft Matter Physics, 2013, 87, pp.041001. 10.1103/PhysRevE.87.041001 · hal-00931429

\section{HAL Id: hal-00931429 \\ https://hal.science/hal-00931429}

Submitted on 11 Apr 2016

HAL is a multi-disciplinary open access archive for the deposit and dissemination of scientific research documents, whether they are published or not. The documents may come from teaching and research institutions in France or abroad, or from public or private research centers.
L'archive ouverte pluridisciplinaire HAL, est destinée au dépôt et à la diffusion de documents scientifiques de niveau recherche, publiés ou non, émanant des établissements d'enseignement et de recherche français ou étrangers, des laboratoires publics ou privés. 


\title{
Turbulence and columnar vortex formation through inertial-wave focusing
}

\author{
Matias Duran-Matute, ${ }^{1, *}$ Jan-Bert Flór, ${ }^{1}$ Fabien S. Godeferd, ${ }^{2}$ and Clément Jause-Labert ${ }^{2}$ \\ ${ }^{1}$ Laboratoire des Écoulements Géophysiques et Industriels, CNRS-Université de Grenoble, Grenoble, France \\ ${ }^{2}$ Laboratoire de Mécanique des Fluides et d'Acoustique, UMR 5509, École Centrale de Lyon, Lyon, France
}

(Received 19 August 2012; published 16 April 2013)

\begin{abstract}
In this experimental and numerical study, we consider the role of inertial waves in the inverse energy cascade and the transfer of momentum in a rotating fluid. An oscillating torus generates two inertial-wave cones with their energy focusing at their apex. For high wave amplitudes, turbulence is generated locally around the focal point, resulting in angular momentum mixing and the generation of a columnar cyclonic vortex. The results suggest that nonlinear dynamics is essential for the wave induced momentum transport towards columnar vortices in rotating turbulence.
\end{abstract}

DOI: 10.1103/PhysRevE.87.041001

PACS number(s): 47.27.-i, 47.32.-y, 47.35.-i

In rotating turbulence, the Coriolis acceleration is responsible for the presence of inertial waves (also known as gyroscopic or Kelvin waves) and the formation of large columnar vortices [1]. The formation of columnar structures is an ever-present feature in rotating turbulent flows that has been observed in flows forced by a continuously oscillating grid [1,2], electromagnetic forcing [3,4], and a translating grid (for decaying flows) [5].

Previously, the formation of these columnar structures was explained using the Taylor-Proudman theorem, but this theorem is strictly valid only for infinite rotation rates and not for turbulent flows. More recent studies have pointed out the importance of inertial waves for this process $[2,5,6]$. Depending on the value of the Rossby number Ro, which represents the ratio between convective and Coriolis acceleration, different regimes have been observed. For strong rotation rates, the columnar structures are seen as zero-frequency inertial waves [2]. For intermediate Ro values, weakly nonlinear triad interactions are responsible for the formation of the columnar vortices [6]. Because of their relevance to momentum transport, inertial waves are at the base of the inverse energy cascade observed in rotating flows, where energy transfers from large to small wave number, which is opposite to the direct energy cascade occurring in three-dimensional turbulence in the absence of background rotation [5].

The aim of the present investigation is to consider the simplest configuration that allows for the study of the origin of vortex-column formation as a function of the Rossby number for sufficiently high Reynolds number Re. With this in mind, we present results from an experimental setup, which differs from previous work in that the turbulent flow is confined to a finite volume within the fluid and originates only from linear inertial waves. Previously, the study of localized turbulence was only possible through numerical simulations.

The well defined turbulent blob was created by the focusing of energy onto the apex of two inertial-wave cones which results in wave breaking for large Re values while the Ro value is kept low. Only the recent advances in particle image velocimetry (PIV) [7], and particularly stereo-PIV (S-PIV),

\footnotetext{
${ }^{*}$ Current address: Department of Physical Oceanography, Royal NIOZ, Den Burg (Texel), The Netherlands.
}

allow for the observation and quantitative study of both inertial-wave beams and the columnar vortex. The experimental results are complemented with results from numerical simulations which show good qualitative and quantitative agreement. In addition, numerical simulations give access to information at scales not resolved in the experiments.

In the current Rapid Communication, we attribute the vortex formation to angular momentum mixing due to the turbulence generated by the inertial-wave breaking. Some applications of this process that have been considered previously are the generations of tornadoes in the atmosphere [8], vortex formation in accretion disks (see Ref. [9] and references therein), and the generation of mean flows in ocean basins by focusing of inertial waves into attractors [10]. The principle of this process is that the horizontal exchange of fluid rings in a rotating fluid gives rise to a cyclonic flow, and at larger radii, anticyclonic flow (see Refs. [8,10,11]).

Experiments were conducted in a cubic tank with sides $100 \mathrm{~cm}$ long. The tank was filled to a depth $H=80 \mathrm{~cm}$ with salt water $\left(\rho=1.02 \mathrm{~kg} \mathrm{~m}^{-3}\right.$ to match the density of particles used for PIV) and placed on top of a rotating table, which rotated at a constant rate $\Omega$. In the center at 40 $\mathrm{cm}$ height above the bottom, a solid Plexiglas torus with a small radius $R=1.5 \mathrm{~cm}$ and a large radius $R_{L}=13.5 \mathrm{~cm}$ oscillated vertically. The oscillation was driven by an electric motor, and the forcing frequency $\sigma$ was controlled through an electric feedback system. We define the coordinate system $\left(\hat{\boldsymbol{e}}_{1}, \hat{\boldsymbol{e}}_{2}, \hat{\boldsymbol{e}}_{3}\right)$ with $\hat{\boldsymbol{e}}_{3}$ parallel to the axis of rotation. The velocity and the vorticity vectors are given by $\boldsymbol{u}=\left(u_{1}, u_{2}, u_{3}\right)$ and $\boldsymbol{\omega}=\left(\omega_{1}, \omega_{2}, \omega_{3}\right)$, respectively.

The torus oscillated as $K \cos (\sigma t)$ with $K$ the amplitude and $0<\sigma<2 \Omega$, generating two inertial-wave cones. According to linear theory, the waves are characterized by the dispersion relation

$$
\cos \alpha=\frac{\sigma}{2 \Omega}
$$

i.e., the angle of propagation of the waves, with respect to the horizontal $\alpha$, is determined by the ratio of the forcing frequency to the rotation frequency. This is similar to the radiation of internal waves in a uniformly stratified fluid. In a vertical section, the energy propagates along rays away from the oscillating torus and forms a pattern similar to the diagonal cross (often called the St. Andrew's Cross) for planar internal 


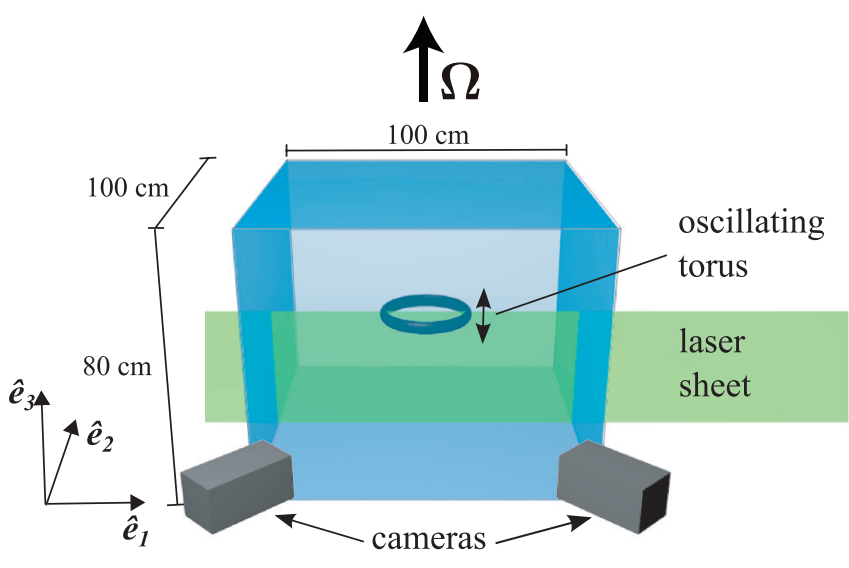

FIG. 1. (Color online) Schematic representation of the experimental setup.

gravity waves [12]. The phase velocity is perpendicular to these rays.

The three velocity components were measured on a vertical plane crossing the center of the torus using S-PIV. For this, the water was seeded with $60 \mu \mathrm{m}$ polymer particles. A vertical laser sheet, of $5 \mathrm{~mm}$ thickness and passing through the center of the ring, illuminated the particles. On one side of the tank, two cameras were positioned, observing the laser plane at angles of $+30^{\circ}$ and $-30^{\circ}$ (see Fig. 1). Images of the illuminated particles were taken with a time interval such that the maximum particle in-plane displacement is smaller than one fourth of the interrogation window size, and the out-of-plane displacement is smaller than one fourth of the laser sheet thickness. For each camera, the particle displacement was obtained by cross correlating two successive images using standard PIV techniques. Later, the in-plane velocity component was obtained through a geometrical reconstruction using the velocity fields from both cameras. Finally, a reconstructed velocity field of about $30 \times 30 \mathrm{~cm}^{2}$ below the torus was obtained. For the S-PIV calculations, the UVMAT/CIV software packages developed at the Coriolis Rotating Platform at the LEGI (Grenoble, France) ${ }^{1}$ were used.

For fixed ratio $R / R_{L}$, there are three nondimensional parameters governing the problem: the Reynolds number,

$$
\operatorname{Re}=\frac{\sigma K(2 R)}{v},
$$

which represents a ratio of convective acceleration to viscous momentum diffusion; the Rossby number

$$
\mathrm{Ro}=\frac{\sigma K}{2 R \Omega}
$$

and the angle of propagation $\alpha$ obtained from the dispersion relation (1). Sometimes, the ratio $\sigma / 2 \Omega$ is referred to as the Rossby number, but since the relative magnitude of convective acceleration with respect to that of the Coriolis acceleration is better represented by (3) for the flow studied, we prefer this definition.

\footnotetext{
${ }^{1}$ Information and code are available at http://coriolis.legi.grenobleinp.fr
}

TABLE I. Parameter values for the experiments and numerical simulations presented.

\begin{tabular}{lcccccc}
\hline \hline Expt. & $\Omega(\mathrm{rad} / \mathrm{s})$ & $\sigma(\mathrm{rad} / \mathrm{s})$ & $\mathrm{K}(\mathrm{cm})$ & $\mathrm{Re}$ & $\alpha$ & $\mathrm{Ro}$ \\
\hline 1 & 0.889 & 1.26 & 0.25 & 94 & $\pi / 4$ & 0.12 \\
2 & 1.110 & 1.56 & 1.00 & 470 & $\pi / 4$ & 0.47 \\
\hline \hline
\end{tabular}

To explore the parameter space, nine different experiments were performed for various values of the frequency $\sigma$, the amplitude $K$ of the oscillation, and the rotation rate of the table $\Omega$. In the current paper, we focus on the results of two of these experiments (see Table I) and their corresponding numerical simulations. These experiments are extreme cases within the parameter space explored: with Expt. 1 having a low Re value and a low Ro value, and hence, being characterized by weak wave-breaking effects due to the wave focusing, and Expt. 2 having the highest Re value for $\mathrm{Ro}<0.5$ and a clearly turbulent focal region. The other experiments represent intermediate situations with only quantitative differences.

Numerical simulations were performed using a pseudospectral code based on Fourier polynomials that solves the full Navier-Stokes equations with a resolution of $576^{3}$ and volume penalization for the moving torus and the upper and lower boundaries (see Ref. [13] for more details). Side boundaries were periodic. In view of the flow symmetry and the weak wave energy near these boundaries, this choice does not affect the results.

Figure 2 shows isosurfaces of the vertical component of the velocity $u_{3}$ for the simulation equivalent to Expt. 1 . The inertial-wave cone excited by the oscillating torus can be clearly observed. Figure 3(a) shows the phase averaged velocity taken at phase $t=2 n \pi$ with $n=0,1,2, \ldots$ and Fig. 3(b) the velocity magnitude averaged over 60 periods, with both figures clearly revealing the wave beams. The wave energy grows from the torus towards the apex of the cones, and it decreases afterwards due to the radial spreading. However, the energy does not increase monotonically towards the apex but it is concentrated within a close to spherical region. The diameter of this region is given by the thickness of the wave beams

$$
\delta=2 R+6.84\left(\frac{v r}{\sigma \tan \alpha}\right)^{1 / 3}
$$

at $r=R_{L} \sin \alpha$, where $r$ is the distance from the center of the tube along a wave beam (see Ref. [14] for details). For this experiment, the average velocity at the apex of the cones

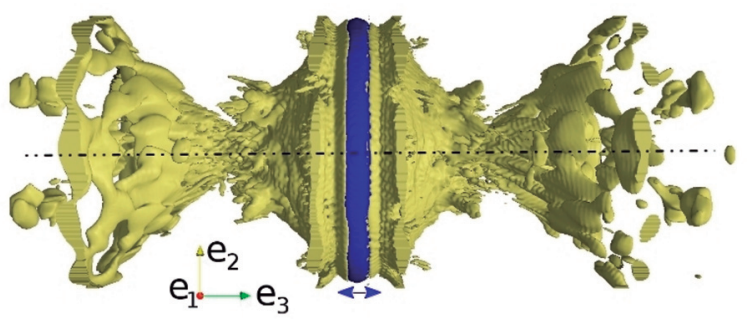

FIG. 2. (Color online) Inertial-wave cones excited by the oscillating torus as depicted by isosurfaces of $u_{3}$ from a numerical simulation equivalent to Expt. 1. The figure has been rotated $90^{\circ}$ for presentation purposes. The torus is shown at the center of the pictures (in blue). 

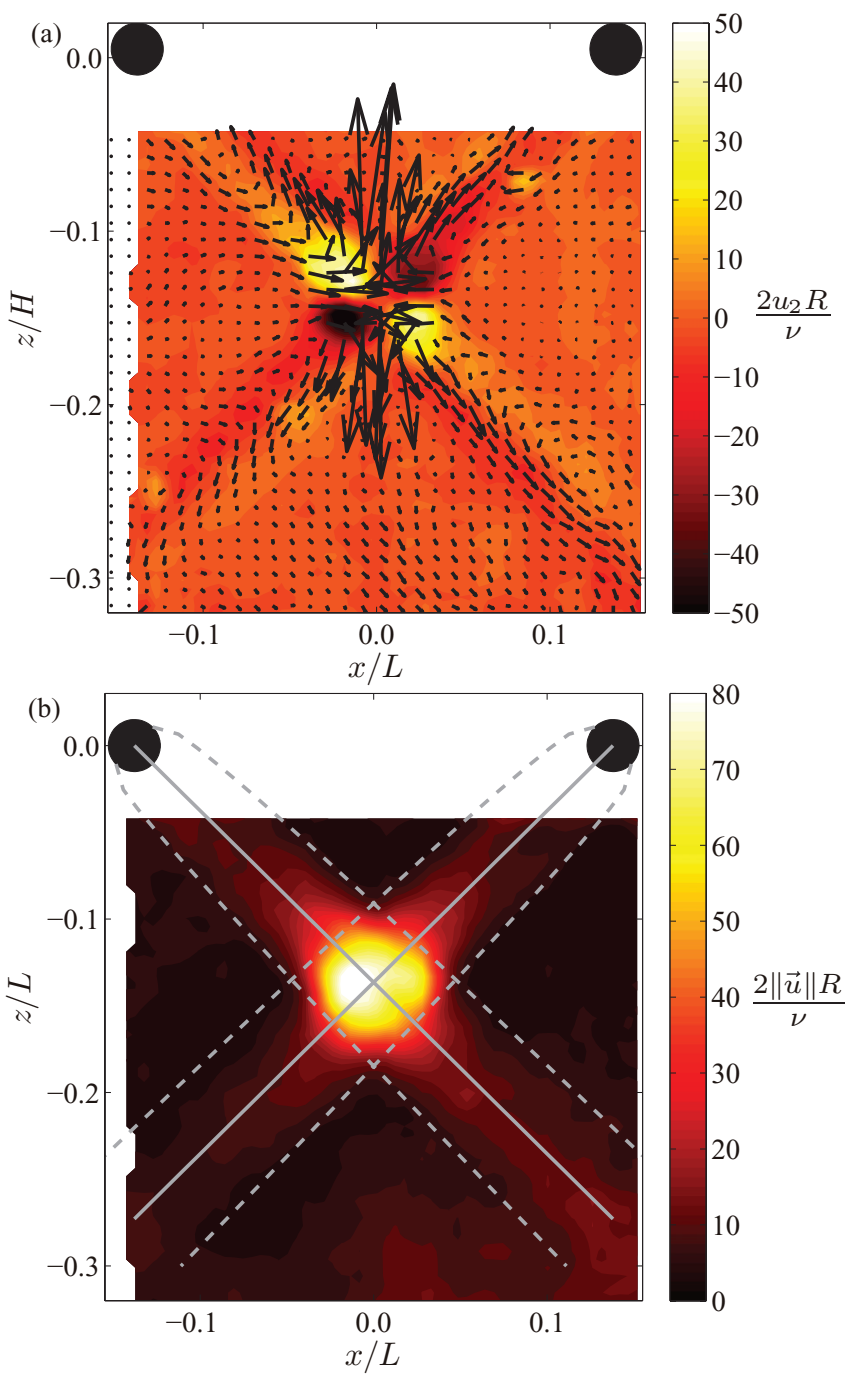

FIG. 3. (Color online) (a) Phase averaged velocity (at phase $t=2 n \pi$ with $n=0,1,2, \ldots)$ for Expt. 1 . Only one in every two vectors obtained using PIV is shown. The color denotes the nondimensional out-of-plane velocity $2 u_{2} R / v$. (b) Nondimensional velocity magnitude averaged over 60 periods for the same experiment. The continuous line represents the center of the wave beam. The dashed line denotes the envelope of the wave beams given by (4). The black circles denote the position of the torus.

is about eight times larger than the velocity along the wave beam closer to the torus.

Figure 4 shows the experimentally (a) and numerically (b) obtained nondimensional time-averaged velocity magnitude for the parameters of Expt. 2. The two (nondimensionalized) results reveal good agreement in velocity magnitude and also in flow structure: The kinetic energy concentrates at the apex of the inertial-wave cones, from which a long vertical cyclonic vortical structure emanates. However, the simulations seem to be less dissipative than the experiments as more energy is found outside the wave cones.

The vortical structure can be clearly observed by averaging either the azimuthal velocity, or the vertical vorticity over an integer number of periods [see Figs. 5(a) and 5(b), respectively]. Turbulence is generated by the breaking of the inertial waves around the apex of the cone and mixes angular
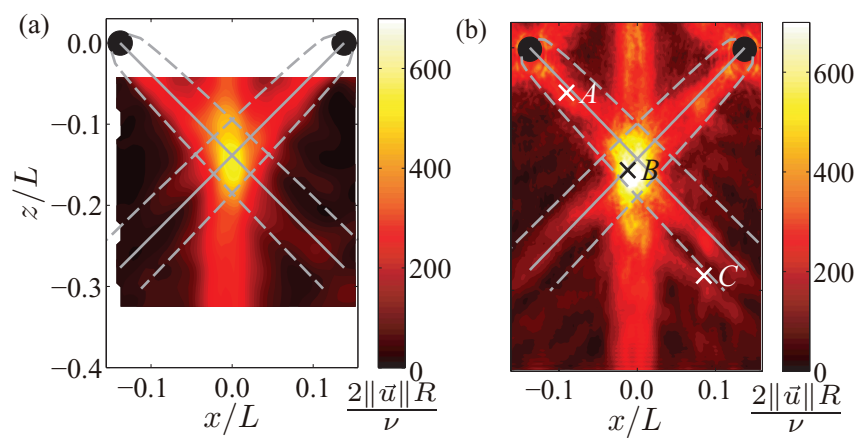

FIG. 4. (Color online) (a) Velocity magnitude averaged over 60 periods for Expt. 2. (b) Velocity magnitude averaged over three periods for the numerical simulation equivalent to Expt. 2. The crosses denote the points at which the temporal spectra is calculated for Fig. 6. Only a part of the numerical domain is shown. The rest is as in Fig. 3.

momentum irreversibly [8]. This explanation is supported by the temporal spectra of the flow. Figure 6 shows power spectral density (PSD) of $u_{3}$ at three different points along the inertial wave beam [their locations are shown in Fig. 4(b)]. The spectra were calculated from the numerical simulations since the experimental measurements have a temporal resolution which is too coarse to resolve the frequencies of the turbulence. For frequencies higher than $2 \Omega$, the spectra quickly decay outside the high-energy region, whereas inside much more energy content is found for these frequencies. This is a clear indication of a more efficient cascade towards higher frequencies inside the blob, and that this nonlinear behavior is confined within the focal region of wave breaking.

The wave beams are much weaker after the apex of the cone since part of their energy is transferred to the vortex (see Fig. 4). The transfer of energy from the waves towards the columnar vortex is determined from the energy of the mean flow by integration of the velocity field over an integer number of forcing periods. The integration filters out the wave motion but retains the mean flow. The experimental and numerical results for Expt. 2, both show that the mean azimuthal flow contains $45 \%$ of the total energy within the experimental measurement area, thus confirming the quantitative agreement between both approaches. The numerics allow therefore to extrapolate and to calculate the energy content of the mean flow in the entire
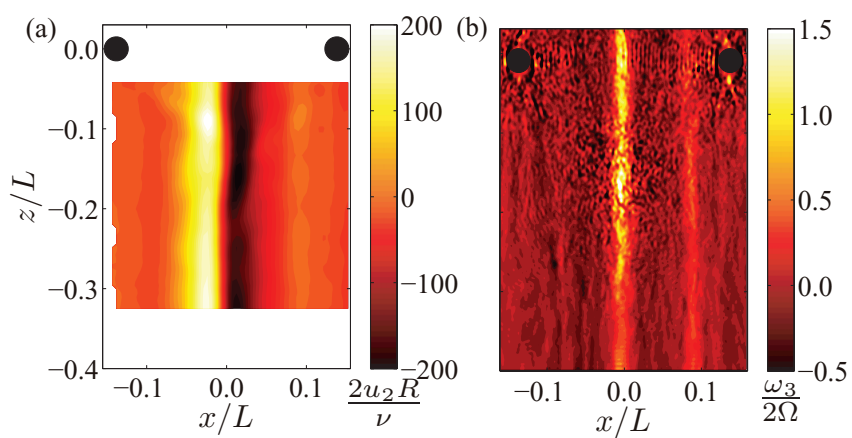

FIG. 5. (Color online) (a) Mean out-of-plane velocity over a 60 forcing periods for Expt. 2. (b) Mean vertical vorticity obtained from the numerical simulation equivalent to Expt. 2 by averaging over three forcing periods. 


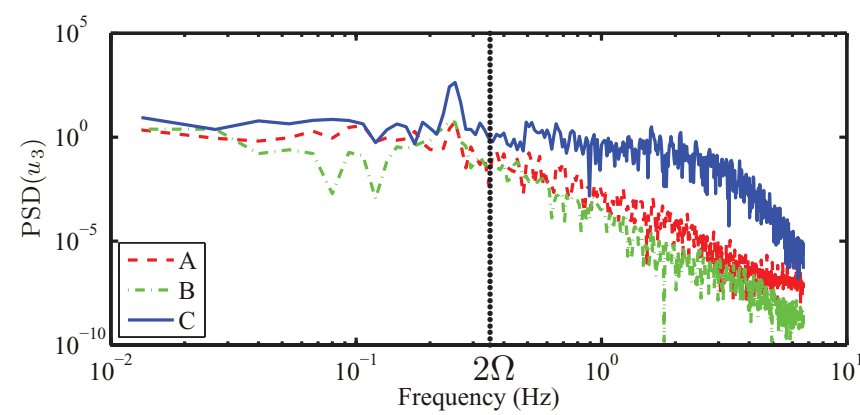

FIG. 6. (Color online) Power spectral density (PSD) of the vertical velocity component $u_{3}$ at points $\mathrm{A}, \mathrm{B}$, and $\mathrm{C}$ of Fig. 4 along the wave beam for the simulation corresponding to Expt. 2 .

tank. This amounts to $30 \%$ of the total energy, in contrast to $13 \%$ for the case of Expt. 1. Angular momentum redistribution that occurs due to wave breaking in the focal region is therefore very efficient in transferring energy towards columnar vortices even for relatively low Re values.

The transfer of energy from inertial waves towards a mean flow is relevant to many geophysical and astrophysical flows. These results on focusing inertial waves show the formation of a mean azimuthal flow due to the redistribution of angular momentum in the wave-breaking region. In addition, different spectral behavior is observed inside and outside the turbulent blob. These experiments allow us, therefore, to further investigate the relevance of the redistribution of angular momentum to the inverse energy cascade in rotating turbulent flows.

We thank Pierre-Alain Barraud for technical support and Leo Maas for fruitful discussions. FG acknowledges the CINES for the use of its computing facilities. This collaborative work was financed by the ANR "ANISO."
[1] E. J. Hopfinger, F. K. Browand, and Y. Gagne, J. Fluid Mech. 125, 505 (1982).

[2] P. J. Staplehurst, P. A. Davidson, and S. B. Dalziel, J. Fluid Mech. 598, 81 (2008).

[3] L. Del Castello and H. J. H. Clercx, Phys. Rev. Lett. 107, 214502 (2011).

[4] L. J. A. van Bokhoven, H. J. H. Clercx, G. J. F. van Heijst, and R. R. Trieling, Phys. Fluids 21, 096601 (2009).

[5] C. Lamriben, P.-P. Cortet, and F. Moisy, Phys. Rev. Lett. 107, 024503 (2011).

[6] L. M. Smith, J. R. Chasnov, and F. Waleffe, Phys. Rev. Lett. 77, 2467 (1996).

[7] L. Messio, C. Morize, M. Rabaud, and F. Moisy, Exp. Fluids 44, 519 (2007).
[8] R. O. R. Y. Thompson, Geophys. Astrophys. Fluid Dyn. 12, 221 (1979).

[9] S. A. Balbus, Annu. Rev. Astron. Astrophys. 41, 555 (2003).

[10] L. R. M. Maas, J. Fluid Mech. 437, 13 (2001).

[11] A. D. McEwan, Geophys. Astrophys. Fluid Dyn. 5, 283 (1973).

[12] D. E. Mowbray and B. S. H. Rarity, J. Fluid Mech. 28, 1 (1967).

[13] C. Jause-Labert, F. Godeferd, and B. Favier, Comput. Fluids 67, 41 (2012).

[14] P.-P. Cortet, C. Lamriben, and F. Moisy, Phys. Fluids 22, 086603 (2010). 